Pacific Journal of Mathematics

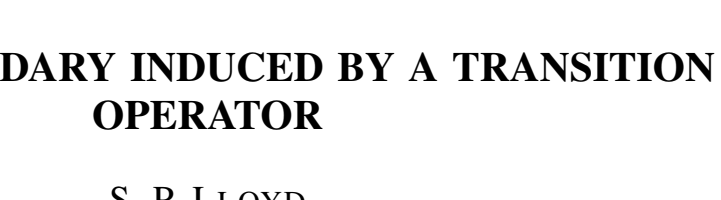




\title{
FELLER BOUNDARY INDUCED BY \\ A TRANSITION OPERATOR
}

\author{
S. P. LLOYD
}

A transition operator $T$ is a nonnegative contraction on an $A L$ space $L$ such that $\|T \mu\|=\|\mu\|$ for $\mu \geqq 0$. The set $\mathscr{L}=\left\{f \in L^{*}: T^{*} f=f\right\}$ of invariant functions of the adojoint $T^{*}$ turns out to be lattice isomorphic to $C(B)$ for a certain hyperstonian compact Hausdorff $B$. For the transition operator of a countable state Markov chain, $B$ is the Feller boundary of the process, and in the general case we call $B$ the Feller boundary induced by $T$. For the general case we exhibit several Markov processes associated with $T$ such that $B$ appears as a subset of the state space. These processes involve the potential theory of $T^{*}$. When $L$ is separable there is a quotient space $B_{0}$ of $B$ and a measure $\mu_{0}$ with $B_{0}$ as closed support such that $\mathscr{C}$ is isomorphic to $L_{\infty}\left(B_{0}, \mu_{0}\right)$. There is also a Markov process whose paths converge to $B_{0}$ with probability 1 . However, we do not obtain the kernel representation of superharmonic functions as with the Martin-Doob boundary.

2. The Feller boundary, Let $L$ be an $A L$ space (abstract $l$ space [3, Ch. VI]) and let $T$ be a bounded linear operator on $L$ with the properties $T \geqq 0,\|T \mu\|=\|\mu\|$ for $\mu \geqq 0$, so that $\|T\|=1$. When $L$ is a space of measures our conditions are that $T$ transform probabilities into probabilities. As we will see, there are various discrete parameter Markov processes associated with $T$ such that $T$ induces the transition probabilities of the Markov processes, and we call $T$ a transition operator. Let $M$ be the Banach space conjugate of $L$, so that $M$ is an $A M$ space with order unit. The adjoint $T^{*}$ is an operator on $M$ such that $T^{*} \geqq 0, T^{*} 1=1,\left\|T^{*}\right\|=1$. Let $\mathscr{L}$ be the subspace of $L$ consisting of the invariant vectors $\mu=T \mu$ of $T$, and let $\mathscr{C l}$ be the subspace of $M$ consisting of the invariant vectors $f=T^{*} f$ of $T^{*}$. We will see that $\mathscr{C}$ corresponds to the space of invariant (or harmonic or regular or concordant) functions of a Markov process, while $\mathscr{L}$ corresponds to a certain closed subspace of the invariant measures of the process. We will be concerned mainly with $\mathscr{l l}$.

Let $X$ be the (Kakutani) space of lattice homomorphisms of $M$ onto the reals, with the topology induced by $M$, so that $M$ is isometrically linearly and lattice isomorphic to real $C(X)$ on hyperstonian compact Hausdorff $X$. From now on we identify $M$ with $C(X)$. We represent $M^{*}$ as the space rca $(X)$ of regular bounded signed Borel measures on $X$. For each $\nu \in$ rea $(X), \mathscr{S}(\nu)$ will denote the closed support of $\nu$. We denote by $\kappa: L \rightarrow \operatorname{rca}(X)$ the natural injection of $L$ into its second 
conjugate space; $\kappa$ is an isometric linear and lattice isomorphism of $L$ onto the set of normal measures on $X$, that is, measures which vanish on nowhere dense sets. From time to time we will consider the case where $L$ has an $F$ unit (weak order unit, Freudenthal unit), that is, an element $\pi \geqq 0$ such that $\pi \wedge|\lambda|=0$ implies $\lambda=0$ for $\lambda \in L$. If $\pi$ is an $F$ unit then $\kappa \pi \in \operatorname{rca}(X)$ is a category measure, that is, $\kappa \pi$ vanishes on nowhere dense sets and is strictly positive on nonempty open sets. In this event, we may identify $\kappa L$ with $L_{1}(X, \kappa \pi)$. (Here and in every other case, if the $\sigma$-field is not mentioned then it is the Borel sets of the compact Hausdorff space involved.)

Our identification of $M$ with $C(X)$ makes $T^{*}: C(X) \rightarrow C(X)$ an operator on $C(X)$ with the properties $T^{*} \geqq 0, T^{*} 1=1,\left\|T^{*}\right\|=1$. Such an operator has a representation by a kernel $t$ :

$$
\left(T^{*} f\right)(x)=\int f\left(x^{\prime}\right) t_{x}\left(d x^{\prime}\right), \quad x \in X, f \in C(X),
$$

where (i) $t_{x} \in \mathrm{rca}(X)$ is a probability measure for each $x \in X$, (ii) the mapping $t: X \rightarrow \mathrm{rca}(X)$ is weakly* continuous. We may regard $t$ as the transition probability of a Feller type Markov process on state space $X$. Only the normal measures will be considered as initial probabilities.

REMARK. If $L$ is given concretely as a space of measures on some measurable space, it may happen that the corresponding kernel on the given space is only finitely additive; the regularization of conditional probabilities by going to a representation space is well known [6].

REMARK. The more general case where only $T \geqq 0,\|T\| \leqq 1$ is assumed can be reduced to the case we treat by the familiar device of adjoining a terminal state; we omit the details.

The second adjoint $T^{* *}$ : rca $(X) \rightarrow \operatorname{rca}(X)$ is given by

$$
T^{* *} \nu=\int t_{x} \nu(d x), \quad \nu \in \operatorname{rca}(X),
$$

where the integration is in the weak* sense. If $\nu$ is normal then $T^{* *} \nu$ is normal; indeed, $T^{* *}(\kappa \mu)=\kappa(T \mu), \mu \in L$. Later on we will need a stronger version of (1), as follows. For each Borel set $E \subset X$ the function $t_{x}(E), x \in X$, is Borel measurable. The integral of this function with respect to $\nu \in \operatorname{rea}(X)$ is

$$
\left(T^{* *} \nu\right)(E)=\int t_{x}(E) \nu(d x), \quad \text { Borel } E \subset X, \nu \in \operatorname{rca}(X)
$$

For proof, see [11, p. 175].

Let $\lambda$ be a Banach limit, considered as a finitely additive shift in- 
variant probability on the field of subsets of the positive integers $N_{+}$. Consider the operator $Q(\lambda): C(X) \rightarrow C(X)$ defined by

$$
\int_{N_{+}}\left(\mu, T^{* n} f\right) \lambda(d n)=(\mu, Q(\lambda) f), \quad \mu \in L, f \in C(X) .
$$

This is construction of Day [2] as applied to the semigroup of additive positive integers acting on $L$, and yields $Q(\lambda)$ as a projection onto the subspace $\mathscr{C}$ of invariant vectors of $T^{*}$, and such that $T^{*} Q(\lambda)=$ $Q(\lambda) T^{*}=Q(\lambda)$. The properties $T^{*} \geqq 0, T^{*} 1=1,\left\|T^{*}\right\|=1$ of $T^{*}$ imply $Q(\lambda) \geqq 0, Q(\lambda) 1=1,\|Q(\lambda)\|=1$. If $\lambda_{1}, \lambda_{2}$ are Banach limits then $Q\left(\lambda_{2}\right) Q\left(\lambda_{1}\right)=Q\left(\lambda_{1}\right)$.

Such a projection has a kernel representation

$$
(Q(\lambda) f)(x)=\int f\left(x^{\prime}\right) q_{x}^{(\lambda)}\left(d x^{\prime}\right), \quad x \in X, f \in(C X),
$$

with each $q_{x}^{(\lambda)} \in \operatorname{rca}(X)$ a probability measure and $q^{(\lambda)}: X \rightarrow \operatorname{rca}(X)$ weakly* continuous. The adjoint is

$$
Q^{*}(\lambda) \nu=\int q_{x}^{(\lambda)} \nu(d x), \quad \nu \in \operatorname{rca}(X) .
$$

Let $\varphi$ be the equivalence in $X$ induced by $\mathscr{C}$, that is, $x_{1} \varphi x_{2}$ if and only if $f\left(x_{1}\right)=f\left(x_{2}\right)$ for all $f \in \mathscr{R}$. Let $Z=X / \varphi$ be the quotient space, let $\theta: X \rightarrow Z$ be the quotient mapping, and for each $x \in X$ let $\mathscr{C}_{x}=$ $\theta^{-1}(\theta x)$ be the $\varphi$ equivalence class containing $x$. The elements of $\mathscr{K}$ drop to $Z$ and give a subspace $\mathscr{C}_{1}$ of $C(Z)$ which separates the points of $Z$. Let $B \subset Z$ be the Choquet boundary of $\mathscr{L}_{1}$ [13]. The existence of the projection $Q(\lambda)$ implies that $B$ is closed, and that $\mathscr{C}$ is isometrically linearly and order isomorphic to $C(B)[13, \S 9]$ [9] [10]. If $x \in \theta^{-1} B$ then $\mathscr{S}\left(q_{x}^{(\lambda)}\right) \subset \mathscr{C}_{x}$, and $\mathscr{S}\left(q_{x}^{(\lambda)}\right) \subset \theta^{-1} B$ for each $x \in X$. Let $\bar{q}: x \rightarrow \operatorname{rca}(B)$ be defined by $\bar{q}_{x}(E)=q_{x}^{(\lambda)}\left(\theta^{-1} E\right), x \in X$, Borel $E \subset B$. The isomorphism of $C(B)$ onto $\mathscr{C}$ is effected by $\Phi: C(B) \rightarrow C(X)$ given by

$$
(\Phi h)(x)=\int h(z) \bar{q}_{x}(d z), \quad x \in X, h \in C(B) .
$$

The adjoint $\Phi^{*}: \operatorname{rca}(X) \rightarrow \operatorname{rca}(B)$ is onto and is given by

$$
\Phi^{*} \nu=\int \bar{q}_{x} \nu(d x), \quad \nu \in \operatorname{rca}(X) .
$$

This may be interpreted as follows. For $\nu \in$ rea $(X)$ the measure $Q^{*}(\lambda) \nu$ has closed support in $\theta^{-1} B$ and is a balayage of $\nu$ onto the Choquet boundary $\theta^{-1} B$ of the subspace $\mathscr{C}$ of $C(X)$. If we restrict the domain of $Q^{*}(\lambda) \nu$ to sets of the form $O^{-1} E$, Borel $E \subset B$, then we obtain the measure $\Phi^{*} \nu \in$ rca $(B)$. As the notation indicates, this measure is independent of the choice of the generating Banach limit $\lambda$. 
On the other hand, the balayage $Q^{*}(\lambda) \nu$ does depend on $\lambda$, in general. If we fix $\lambda$ then the set $\left\{q_{x}^{(\lambda)}: x \in \theta^{-1} B\right\}$ with the relative weak* topology is a copy of $B$ in $\operatorname{rca}(X)$; a different choice of $\lambda$ will give a different copy of $B$.

Consider now the subspace $\mathscr{L}$ of invariant vectors of $T$. If $\mu \in \mathscr{L}$ then it is straightforward from (2) that $Q^{*}(\lambda) \kappa \mu=\kappa \mu$. Let $\mathscr{S}(\mathscr{L})$ denote the closure of $U\{\mathscr{S}(\kappa \mu): \mu \in \mathscr{L}\}$. Since each $\mathscr{S}(\kappa \mu)$ is open, $\mathscr{S}(\mathscr{L})$ is open. The normal measures with closed support in $\mathscr{S}(\mathscr{L})$ constitute the norm closed order ideal subspace $\kappa \mathscr{L}_{1}$ generated by $\mathscr{L}$. It follows from results of Sucheston [14], the author [10], and Namioka [12] that if $\mu \in \mathscr{L}_{1}$ then the ergodic averages $n^{-1} \sum_{1}^{n} T^{j} \mu$ are convergent in norm to $\nu \in \mathscr{L}$, with $Q^{*}(\lambda) \kappa \mu=\kappa \nu$ independent of the choice of $\lambda$. Moreover, $\kappa \mu$ is $\kappa \nu$-continuous. It is easy to see that for $x \in \mathscr{S}(\mathscr{L}), q_{x}^{(\lambda)}$ is independent of $\lambda$. (Let $\kappa \mu_{\alpha} \rightarrow \delta_{x}$ weakly*, with $\mathscr{S}\left(\kappa \mu_{\alpha}\right) \subset \mathscr{S}\left(\mathscr{Q}^{P}\right)$ and $\delta_{x} \in \mathrm{rca}(X)$ the evaluation measure at $x$. By what has been said above, $Q^{*}(\lambda) \kappa \mu_{\alpha}$ is independent of $\lambda$, as is then $\lim _{\alpha} Q^{*}(\lambda) \kappa \mu_{\alpha}=Q^{*}(\lambda) \delta_{x}=q_{x}^{(\lambda)}$.) On the other hand, suppose $x \in X$ is such that $q_{x}^{(\lambda)}$ is independent of $\lambda$ on a neighborhood of $x$. With $x \in \mathscr{S}(\kappa \mu)$ a subset of this neighborhood, $Q^{*}(\lambda) \kappa \mu$ is independent of $\lambda$, the ergodic averages of $\kappa \mu$ are convergent, $Q^{*}(\lambda) \kappa \mu$ is normal and hence $Q^{*} \kappa \mu \subset \kappa \mathscr{L}$. It must be the case then that $\mathscr{S}\left(q_{x}^{(2)}\right) \subset \mathscr{S}(\mathscr{P})$ for such $x$.

We turn now to the subspace $\mathscr{C}$ of invariant vectors of $T^{*}$. The isomorphism $\Phi$ of $C(B)$ onto $\mathscr{M}$ serves to lift the lattice and algebraic operations of $C(B)$ to $\mathscr{C}$. It is easy to see that the $\mathscr{M}$ supremum and $\mathscr{C}$ product of $f, g \in \mathscr{C}$ are given by $Q(\lambda)(f \vee g)$ and $Q(\lambda)(f \cdot g)$, respectively, where in the parentheses the $C(X)$ (pointwise) operations are intended. These operations do not depend on the choice of $\lambda$. The lattice and algebraic operations in $\mathscr{C}$ coincide with those of $C(X)$ only when $\mathscr{C l}$ is a (pointwise) subalgebra of $C(X)$.

Let $\mathscr{N}$ be the norm closure of the subspace $\{\mu-T \mu: \mu \in L\}$. It is straightforward that $\mathscr{N}=\mathscr{C}_{\perp}$ is the weak* annihilator of $\mathscr{M}$, and that $\mathscr{C}=\mathscr{N}^{\perp}=\left(\mathscr{M}_{\perp}\right)^{\perp}$ is weakly* closed. Let $\mathscr{R}$ be the quotient space $\mathscr{R}=L / \mathscr{N}$, with elements consisting of equivalence classes $\mu+\mathscr{N}, \mu \in L$. The Banach space conjugate $\mathscr{R}^{*}$ is isometrically linearly isomorphic to $\mathscr{M}$. We give $\mathscr{R}$ the order induced by $\mathscr{H}$, and observe that the quotient mapping $\mu \rightarrow \mu+\mathscr{N}$ is order preserving.

THEOREm 1. Space $B$ is hyperstonian, and $\mu+\mathscr{N}^{-} \rightarrow \Phi^{*} \kappa \mu$ is an isometric linear and order isomorphism of $\mathscr{R}$ onto the space of all normal measures on $B$.

Proof. We prove first that $B$ is extremally disconnected. Let 
$\left\{u_{\alpha}\right\}$ be any collection of members of $\mathscr{C l}$ bounded below. Since $C(X)$ is a complete lattice, the infimum $v=\wedge u_{\alpha}$ exists in $C(X)$. Consider $w=Q(\lambda) v$; we will show that $w$ is the infimum of $\left\{u_{\alpha}\right\}$ in $\mathscr{C}$. First, note that $v \leqq u_{\alpha}$ and $Q(\lambda) \geqq 0$ imply $w=Q(\lambda) v \leqq Q(\lambda) u_{\alpha}=u_{\alpha}$. Suppose $h \in \mathscr{l l}$ is such that $h \leqq u_{\alpha}$ for all $\alpha$. Then $h \leqq v$ in $C(X)$ and hence $h=Q(\lambda) h \leqq Q(\lambda) v=w$. It follows that $w$ is the infimum of $\left\{u_{\alpha}\right\}$ in $\mathscr{C}$. Since $\mathscr{C}$ is lattice isomorphic to $C(B)$, space $B$ is extremally disconnected.

Let $\left\{u_{\alpha}\right\}$ be a monotone decreasing generalized sequence in $C(B)$ with infimum $u$ in the complete latice $C(B)$. Because $\Phi$ is order preserving, $\left\{\Phi u_{\alpha}\right\}$ is a monotone decreasing generalized sequence in $C(X)$ bounded below by $\Phi u$. Let $v$ be the infimum of $\left\{\Phi u_{\alpha}\right\}$ in the complete lattice $C(X)$. The measures $\kappa L$ are normal on $X$, so that $\lim _{\alpha}\left(\Phi \mu_{\alpha}, \kappa \mu\right)=$ $(v, \kappa \mu)$ for each $\mu \in L$. In particular, $0=\lim _{\alpha}\left(\Phi u_{\alpha}, \kappa, \mathcal{N}\right)=(v, \kappa \mathcal{N})$, whence $v \in . / l$ and $\Phi u=Q(\lambda) v=v$. Thus for each $\mu \in L$ we have $\lim _{\alpha}\left(u_{\alpha}, \Phi^{*} \kappa \mu\right)=\lim _{\alpha}\left(\Phi u_{\alpha}, \kappa \mu\right)=(\Phi u, \kappa \mu)=\left(u, \Phi^{*} \kappa \mu\right)$, and it follows that $\Phi^{*} \kappa \mu$ is normal on $B$. Now, $\Phi^{*} \kappa \mathscr{N}=0$ and $\mathscr{C}=$ range $\Phi$ is total on $\mathscr{R}$, so that $\mu+\mathscr{N} \rightarrow \Phi^{*} \kappa \mu$ is an isometric linear and order isomorphism of $\mathscr{R}$ onto a norm closed subspace of the space of normal measures on $B$. Because $C(B)$ is isomorphic to $\mathscr{K}^{*}$, this subspace is total on $C(B)$; a fortiori, the normal measures on $B$ are total on $C(B)$. This is to say, $B$ is hyperstonian. It is known that when $B$ is hyperstonian then $C(B)$ is the Banach space conjugate of the space of normal measures on $B$, and it is straight-forward that $\mu+\mathscr{N} \rightarrow \Phi^{*} \kappa \mu$ is an isomorphism of $\mathscr{R}$ onto the set of all normal measures on $B$.

Under the isomorphism, $\mathscr{P}$ becomes a complete lattice; in fact, an $A L$ space.

Corollary. If $L$ has an $F$ unit $\pi$ then $\Phi^{*} \kappa \pi$ is a category measure for $B$.

Proof. A normal measure $\nu \geqq 0$ on an extremally disconnected compact Hausdorff space $W$ is a category measure if and only if $(f, \nu)>0$ for every $f \geqq 0, f \neq 0, f \in C(W)$. Suppose $L$ has an $F$ unit $\pi$, so that $\kappa \pi$ is a category measure for $X$. If $u \in C(B)$ is such that $u \geqq 0, u \neq 0$, then $\Phi u \in C(X)$ is such that $\Phi u \geqq 0, \Phi u \neq 0$, whence $\left(u, \Phi^{*} \kappa \pi\right)=(\Phi u, k \pi)>0$. Thus $\Phi^{*} k \pi$ is a category measure for $B$.

Theorem 1 and corollary generalize the result of Kendall [8] for Markov chains.

We now describe a few properties of the representing measures $q_{x}^{(\lambda)}, x \in \theta^{-1} B$. These properties have their roots in the recurrence behavior of the Markov process. It is not our intention to give a detailed investigation of the Markov process, however, and our argu- 
ments are entirely analytical and topological.

Fix $x \in \theta^{-1} B$; the equivalence class $\mathscr{C}_{x}$ is a closed set in the extremally disconnected space $X$, and so consists of an open closed interior and a disjoint closed nowhere dense boundary. Suppose int $\mathscr{C}_{x} \neq \varnothing$. Then there exists a normal probability measure $\kappa \mu$ such that $\mathscr{S}(\kappa \mu) \subset \operatorname{int} \mathscr{C}_{x}$. The members of $\mathscr{C}$ are constant on $\mathscr{C}_{x}$, whence $(\Phi u, \kappa \mu)=u(\theta x), u \in C(B)$, and there follows $\Phi^{*} \kappa \mu=\delta_{\theta x}$. By Theorem $1, \Phi^{*} \kappa \mu$ is a normal measure on $B$, with closed support which is open. This is to say, $\theta x$ is an open point of $B$. We have proved:

Theorem 2. For $x \in \theta^{-1} B$, if $\mathscr{C}_{x}$ has nonempty interior then $\theta x$ is an isolated point of $B$. We remark that if $L$ has an $F$ unit $\pi$ then $\Phi^{*} \kappa \pi$ gives positive measure to every isolated point of $B$, so that the number of isolated points is at most countable.

Fix $x \in \theta^{-1} B$, and decompose $q_{x}^{(2)}=\nu^{\prime}+\nu^{\prime \prime}$ into a normal part $\nu^{\prime}$ and a part $\nu^{\prime \prime}$ whose closed support is nowhere dense. From $T^{* *} q_{x}^{(\lambda)}=$ $q_{x}^{(\lambda)}$ we obtain $T^{* *} \nu^{\prime}-\nu^{\prime}=\nu^{\prime \prime}-T^{* *} \nu^{\prime \prime}$. Now, $T^{* *} \nu^{\prime}$ is normal since $\nu^{\prime}$ is normal, and vanishes on nowhere dense sets. Testing the right hand side above with nowhere dense sets we find $T^{* *} \nu^{\prime \prime}=\nu^{\prime \prime}$ and hence $T^{* *} \nu^{\prime}=\nu^{\prime}$. Thus $\nu^{\prime} \in \kappa \mathscr{L}$, so that $Q^{*}(\lambda) \nu^{\prime}=\nu^{\prime}$. However, $\mathscr{S}\left(\nu^{\prime}\right) \subset \mathscr{C}_{x}$, so that $Q^{*}(\lambda) \nu^{\prime}=\left\|\nu^{\prime}\right\| q_{x}^{(\lambda)}$, by (3). There follows $\nu^{\prime}=$ $\left\|\nu^{\prime}\right\|\left(\nu^{\prime}+\nu^{\prime \prime}\right)$; either $\nu^{\prime}=0$, or $\left\|\nu^{\prime}\right\|=1$ and $\nu^{\prime \prime}=0$. We have proved:

Theorem 3. For $x \in \theta^{-1} B$, either $\mathscr{S}\left(q_{x}^{(\lambda)}\right)$ is nowhere dense or $q_{x}^{(2)}$ is normal.

We observe that when $q_{x}^{(\lambda)}$ here is normal then $x \in \mathscr{S}(\mathscr{L})$, and $\theta x$ is an isolated point of $B$. With $q_{x}^{(\lambda)}$ normal, $\mathscr{C}_{x}$ is a recurrence class for the Markov process, and $q_{x}^{(\lambda)}$ (independent of $\lambda$ ) is the ergodic normal invariant probability for $\mathscr{C}_{x}$.

3. Sojourn solutions. Let $\left\{x_{0}(\omega), x_{1}(\omega), \cdots\right\}$ be the random variables of a Markov process with $t$ as transition probability. If $A \subset X$ is open closed then Prob $\left\{x_{n}(\omega) \in A^{\prime}\right.$ for at most finitely many $\left.n \mid x_{0}(\omega)=x\right\}, x \in X$, differs from a function $s_{A} \in \mathscr{C l}$ on a nowhere dense set. In the terminology of Feller, if $A$ is such that $s_{A} \not \equiv 0$ then $s_{A}$ is a sojourn solution (of $T^{*} f=f$ ). The arguments of Feller show that the sojourn solutions are the extreme points of $\mathscr{C} \cap\{0 \leqq f \leqq 1\}$, and correspond one-to-one to the open closed subsets of $B$. Let us trace this connection in our setup.

Let $W^{A}: C(X) \rightarrow C(X)$ be defined by $W^{A} f=\chi_{A} T^{*}\left(\chi_{A} f\right), f \in C(X)$; the kernel of $W^{A}$ is $w_{x}^{A}(E)=\chi_{A}(x) t_{x}(E \cap A)$, Borel $E \subset X$. We may regard $w^{A}$ as the transition subprobability of the process obtained by 
terminating the $t$ process as soon as it leaves $A$. If $w_{n}=\left(W^{A}\right)^{n} 1$, then $w_{n}(x)$ is the probability that the $t$ process started at $x$ has started in $A$ and remained in $A$ for $n$ transitions. Clearly, $w_{1} \geqq w_{2} \geqq \cdots$, and we let $w$ denote the infimum of $\left\{w_{n}\right\}$ in the complete lattice $C(X)$. For every normal probability $\kappa \mu$ we have $(w, \kappa \mu)=\lim _{n}\left(w_{n}, \kappa \mu\right)$, so that $(w, \kappa \mu)$ is the probability that the process started with $\kappa \mu$ will always be in $A$. From this it is clear that $w \leqq T^{*} w \leqq \cdots$; the lattice supremum is the sojourn solution $s_{A}$. The methods of Feller [5] show that there is an open closed subset $A^{\wedge}$ of $B$ such that $s_{A}=\Phi \chi_{A^{\wedge}}$. Since $s_{A}=Q(\lambda) w \geqq w$ and $Q(\lambda)\left(s_{A}-w\right)=0$, it must be the case that $w=s_{A}$ on $\mathscr{S}\left(q_{x}^{(\lambda)}\right)$ for each $x \in \theta^{-1} B$. Thus the set $\{w>0\}$ either contains $\mathscr{S}\left(q_{x}^{(\lambda)}\right)$ or misses it entirely, and $A^{\wedge}=B \cap \theta(\{w>0\})$. Suppose $A$ is such that $T^{*} \chi_{A} \geqq \chi_{A}$; that is, the process started in $A$ is sure to remain in $A$. Then $w=\chi_{A}$, and $A^{\wedge}=B \cap \theta(A)$.

Let $C$ be an open closed subset of $B$. Put $u_{C}=\Phi \chi_{C}$, and $C^{\sim}=$ $\left\{u_{c}=1\right\}, C^{\vee}=\operatorname{int} C^{\sim}$. From

$$
u_{C}(x)=\int u_{c}\left(x^{\prime}\right) t_{x}\left(d x^{\prime}\right), \quad x \in X,
$$

and $0 \leqq u_{C} \leqq 1$ it follows that if $x \in C^{\sim}$ then $\mathscr{S}\left(t_{x}\right) \subset C^{\sim}$, whence $t_{x}\left(C^{\sim}\right) \geqq \chi_{c^{\sim}}, x \in X$. For all $\mu \geqq 0, \mu \in L$ we have

$$
\left(T^{* *} \kappa \mu\right)\left(C^{\sim}\right)-(\kappa \mu)\left(C^{\sim}\right) \geqq 0
$$

and hence $\left(T \mu-\mu, \chi_{C^{\vee}}\right) \geqq 0$, since $\chi_{C^{\sim}}$ and $\chi_{C^{\vee}}$ have the same integral over normal measures. There follows $\chi_{C^{\vee}} \leqq T^{*} \chi_{C^{\vee}} \leqq \cdots \leqq Q(\lambda) \chi_{C^{\vee}}$. From $\chi_{C^{\vee}} \leqq u_{C}$ we have $Q(\lambda) \chi_{C^{\vee}} \leqq u_{C}=\Phi \chi_{C}$ and hence $\left(C^{\vee}\right)^{\wedge} \subset C$. Let us prove the opposite inclusion. Put $\mathscr{J}_{C}=\{\mu \in L: \kappa \mu$ is a probability such that $\left.\mathscr{S}\left(\Phi^{*} \kappa \mu\right) \subset C\right\}$. For $\mu \in \mathscr{I}_{C}$ we have $1=\left(\chi_{C}, \Phi^{*} \kappa \mu\right)=\left(u_{C}, \kappa \mu\right)$, which requires $\mathscr{S}(\kappa \mu) \subset C^{\vee}, \kappa \mu$ being normal. Thus

$$
1=\left(\chi_{C^{\vee}}, \kappa \mu\right) \leqq\left(Q(\lambda) \chi_{C^{\vee}}, \kappa \mu\right)=\left(\chi_{\left(C^{\vee}\right) \wedge}, \Phi^{*} \kappa \mu\right)
$$

for all $\mu \in \mathscr{I}_{C}$. This implies $\left(C^{\curlyvee}\right)^{\wedge} \supset C$, so we have finally $C=\left(C^{\curlyvee}\right)^{\wedge}$. Thus for each open closed $C \subset B$ we may exhibit $\Phi \chi_{C}$ as a sojourn solution $s_{C^{\vee}}$.

4. Quotient processes. Up to this point the raison d'être for the Feller boundary $B$ is the isomorphism of $\mathscr{C l}$ and $C(B)$. A more direct connection with the state space of a Markov process would be of interest, however. What we seek is a Markov process for which $B$ is a subset of the state space. The quotient space $Z$ of $\theta: X \rightarrow Z$ contains $B$ as a subset, but $T^{*}$ does not drop to $C(Z)$, in general, so $Z$ will not do. We look for an intermediate space $X \rightarrow Y \rightarrow Z$ such that $T^{*}$ drops to $C(Y)$ and the projection measures $q_{x}^{(\lambda)}, x \in \theta^{-1} B$, drop 
to (distinct) evaluations on $Y$. Then the kernel of the dropped $T^{*}$ will be a transition probability on $Y$ and the Choquet boundary of the dropped $\mathscr{C}$ in $Y$ will be a copy of the Feller boundary $B$.

Dually, we find $\mathscr{V}^{n} \subset C(X)$ with the properties

(i ) $\mathscr{V}^{-}$is a closed subalgebra of $C(X)$,

(ii) if $f \in \mathscr{V}^{*}$ then $T^{*} f \in \mathscr{V}^{-}$,

(iii) $\mathscr{C} \subset \mathscr{V}^{n}$,

(iv) for some $\lambda$, if $f \in \mathscr{V}^{-}$then for each $x \in \theta^{-1} B, f$ is constant on $\mathscr{S}\left(q_{x}^{(\lambda)}\right)$.

A $\mathscr{Y}$ satisfying (i)-(iv) will be called suitable. It is clear that the intersection of all suitable $\mathscr{V}^{-}$is the smallest suitable $\mathscr{V}^{\prime}$. As it turns out, for each choice of $\lambda$ there is also a largest suitable $\mathscr{V}$.

Some general considerations first. Let $\mathscr{V}^{-}$be suitable. The constants are in $\mathscr{C l}$, so $\mathscr{V}^{\prime}$ contains the constants. Thus $\mathscr{V}^{\prime}$ is isometrically algebraically isomorphic to $C(Y)$, where $Y$ is the quotient v: $X \rightarrow Y$ of the equivalence in $X$ determined by $\mathscr{Y}$. With $\Theta: C(Y) \rightarrow$ $C(X)$ the induced injection onto $\mathscr{V}^{*}$, the adjoint $\Theta^{*}: \operatorname{rca}(X) \rightarrow \operatorname{rca}(Y)$ maps the normal measures on $X$ onto a closed subspace in rca $(Y)$ which we shall call the special measures. The special measures are total on $C(Y)$, but they will not be normal, in general, and $Y$ is not necessarily hyperstonian. If $\nu_{1} \ll \nu_{2}$ in rea $(X)$ then it is easy to see that $\Theta^{*} \nu_{1} \ll \Theta^{*} \nu_{2}$, so that $\Theta^{*} L_{1}(X, \nu) \subset L_{1}\left(Y, \Theta^{*} \nu\right), \nu \in$ rca $(X)$. On the other hand, $\left\{f \cdot \Theta^{*} \nu: f \in C(Y)\right\}$ is dense in $L_{1}\left(Y, \Theta^{*} \nu\right)$, and since $f \cdot \Theta^{*} \nu=$ $\Theta^{*}(\Theta f \cdot \nu)$ and $\Theta f \cdot \nu \in L_{1}(X, \nu)$, it follows that $\Theta^{*} L_{1}(X, \nu) \rightarrow L_{1}\left(Y, \Theta^{*} \nu\right)$ is onto. (By $f \cdot \mu$ we mean the measure with Radon-Nikodym derivative $d(f \cdot \mu) / d \mu=f$.)

From condition (ii), $T^{*}$ drops to an operator $S: C(Y) \rightarrow C(Y)$ with the properties $S \geqq 0, S 1=1$; the kernel of $S$ is the transition probability of a Feller process on $Y$. From $\Theta S=T^{*} \Theta$ we have $S^{*} \Theta^{*}=$ $\Theta^{*} T^{* *}$, and one sees that $S^{*}$ sends special measures into special measures. It is not necessarily the case that $S$ is an adjoint, however.

From condition (iii), $\mathscr{C l}_{0}=\{f \in C(Y): S f=f\}$ is isomorphic to $\mathscr{C}$. Moreover, for every Banach limit $\lambda^{\prime}, Q\left(\lambda^{\prime}\right)$ drops to a projection $Q_{0}\left(\lambda^{\prime}\right)$ in $C(Y)$ onto $\mathscr{C}_{0}$. By condition (iv), if $x \in \theta^{-1} B$ and $\lambda^{\prime}=\lambda$ then $\Theta^{*} q_{x}^{(\lambda)}=q_{0 y}^{(\lambda)}=\delta_{y}$ with $y=v x$; the set of such $y$ is the Choquet boundary of $\mathscr{C}_{0}$ and is a copy of the Feller boundary $B$ in $Y$. If $\mu \in \operatorname{rca}(Y)$ is a special measure then $\mathscr{S}\left(Q_{0}^{*}(\lambda) \mu\right) \subset B \subset Y$, and $Q_{0}^{*}(\lambda) \mu$ is a normal measure on $B$ in its relative topology. (However, $Q_{0}^{*}(\lambda) \mu$ need not be normal on $Y$; indeed, $B$ may be nowhere dense in $Y$.)

THEOREM 4. For given $\lambda$, the set $\mathscr{V}_{\lambda}^{\prime}=\{f \in C(X)$ : for each $x \in \theta^{-1} B, f$ is constant on $\left.\mathscr{S}\left(q_{x}^{(\lambda)}\right)\right\}$ is the largest suitable $\mathscr{V}$. A characterization is $\mathscr{V}_{\lambda}^{\prime}=\{f \in C(X): Q(\lambda)(f g)=Q(\lambda)[Q(\lambda) f \cdot Q(\lambda) g]$ for every $g \in C(X)\}$ in terms of a weak averaging identity. 
Proof. It is straightforward that condition (i) is satisfied. Each $f \in \mathscr{C}$ is constant on each $\mathscr{C}_{x}$, a fortiori on $\mathscr{S}\left(q_{x}^{(\lambda)}\right) \subset \mathscr{C}_{x}, x \in \theta^{-1} B$, so that condition (iii) is satisfied. From $Q^{*}(\lambda)=Q^{*}(\lambda) T^{* *}$ we have

$$
q_{x}^{(2)}=\int t_{x^{\prime}} q_{x}^{(\lambda)}\left(d x^{\prime}\right), \quad x \in X,
$$

form which it should be clear that $\mathscr{S}\left(t_{x^{\prime}}\right) \subset \mathscr{S}\left(q_{x}^{(2)}\right)$ for $x^{\prime} \subset \mathscr{S}\left(q_{x}^{(\lambda)}\right)$. If $f$ is constant on $\mathscr{S}\left(q_{x}^{(\lambda)}\right)$ then $\left(T^{*} f\right)\left(x^{\prime}\right)=\int f\left(x^{\prime \prime}\right) t_{x^{\prime}}\left(d x^{\prime \prime}\right)$ is constant for $x^{\prime} \in \mathscr{S}\left(q_{x}^{(\lambda)}\right)$. In other words, $T^{*} f \in \mathscr{V}_{\lambda}^{\prime}$ if $f \in \mathscr{V}_{\lambda}^{\prime \prime}$, and condition (ii) is satisfied.

If $f \in \mathscr{V}_{\lambda}^{\prime \prime}$ then $f-Q(\lambda) f=0$ on $\mathscr{S}\left(q_{x}^{(\lambda)}\right)$ for every $x \in \theta^{-1} B$, whence $Q(\lambda)[g(f-Q(\lambda) f)]=0$ for every $g \in C(X)$. Conversely, if $Q(\lambda)[g(f-Q(\lambda) f)]=0$ for every $g \in C(X)$ then $Q(\lambda)\left[(f-Q(\lambda) f)^{2}\right]=0$, which requires $f-Q(\lambda) f=0$ on $\mathscr{S}\left(q_{x}^{(\lambda)}\right)$ for all $x \in \theta^{-1} B$ and thus $f \in \mathscr{V}_{\lambda}^{\prime}$. Since $Q(\lambda)[g Q(\lambda) f]=Q(\lambda)[Q(\lambda) g \cdot Q(\lambda) f]$, we have shown that $\mathscr{V}_{\lambda}^{\prime \prime}=\{f \in C(X): Q(\lambda)(f g)=Q(\lambda)[Q(\lambda) f \cdot Q(\lambda) g]$ for every $g \in C(X)\}$.

The subalgebra $\mathscr{V}_{\lambda}^{\prime}$ depends on the choice of $\lambda$. A smaller subalgebra is obtained by requiring that (iv) hold for every $\lambda$.

Theorem 5. $\mathscr{Y}^{\prime \prime}=\left\{f \in C(X)\right.$ : for each $\lambda$ and each $x \in \theta^{-1} B, f$ is constant on $\left.\mathscr{S}\left(q_{x}^{(\lambda)}\right)\right\}$ is suitable. Equivalent characterizations are $\mathscr{V}^{\prime \prime}=\left\{f \in C(X):\right.$ for each $x \in \theta^{-1} B, f$ is constant on $\left.\bigcup_{\lambda} \mathscr{S}\left(q_{x}^{(\lambda)}\right)\right\}$ and $\mathscr{V}^{\prime \prime \prime}=\{f \in C(X): Q(\lambda)(f g)=Q(\lambda)[Q(\lambda) f \cdot Q(\lambda) g]$ for every $g \in C(X)$ and every Banach limit $\lambda\}$.

Proof. It is clear from $\mathscr{V}^{\prime \prime \prime}=\bigcap_{\lambda} \mathscr{V}_{\lambda}^{\prime \prime}$ that $\mathscr{V}^{\prime \prime \prime}$ is suitable. Now, $Q\left(\frac{1}{2} \lambda_{1}+\frac{1}{2} \lambda_{2}\right)=\frac{1}{2} Q\left(\lambda_{1}\right)+\frac{1}{2} Q\left(\lambda_{2}\right)$, and one sees that if $f \in \mathscr{V}^{\prime \prime \prime}$ then $f$ is constant on $\mathscr{S}\left(q_{x}^{\left(\lambda_{1}\right)}\right) \cup \mathscr{S}\left(q^{\left(\lambda_{2}\right)}\right)$ for every pair $\lambda_{1}, \lambda_{2}$ and hence on $\cup_{\lambda} \mathscr{S}\left(q_{x}^{(\lambda)}\right)$, and this for each $x \in \theta^{-1} B$. The third characterization of $\mathscr{V}^{\prime \prime}$ is clear from Theorem 4.

If $f \in \mathscr{V}^{\prime \prime \prime}$ then for $x \in \theta^{-1} B$ the constant value of $f$ on $\mathscr{S}\left(q_{x}^{(\lambda)}\right)$ is necessarily $(Q(\lambda) f)(x)$, and this must be independent of $\lambda$. This is to say, if $f \in \mathcal{Y}^{\prime \prime \prime}$ then $\left\{T^{* n} f\right\}$ is weakly* almost convergent, in the sense that for each $\mu \in L,\left\{\left(\mu, T^{* n} f\right)\right\}$ is an almost convergent real sequence. It follows that the dropped projection $Q_{0}$ on $C(Y)$ is independent of $\lambda$, and for each $f \in C(Y)$ and special measure $\nu,\left\{\left(S^{n} f, \nu\right)\right\}$ is almost convergent to value $\left(Q_{0} f, \nu\right)$.

We say that $f \in C(X)$ is a weak* potential if $\left\{T^{* n} f\right\}$ is weakly* convergent. The familiar considerations of the Riesz theorem [11, p. 179] show that a weak* potential $f$ is the sum $f=N u+f_{0}$ of a pure potential

$$
N u=w^{*}-\lim \left(1+T^{*}+\cdots+T^{* n}\right) u,
$$


the charge necessarily being $u=\left(1-T^{*}\right) f$, and an invariant function

$$
f_{0}=w^{*}-\lim T^{* n} f=Q(\lambda) f,
$$

necessarily independent of $\lambda$. The set of weak* potentials is a norm closed subspace of $C(X)$; a suitable pseudonorm is

$$
p(f)=\left\|\operatorname{Lim} \operatorname{Sup} T^{* n} f-\operatorname{Lim} \operatorname{Inf} T^{* n} f\right\|,
$$

with $\operatorname{Lim} \operatorname{Sup}=\mathbb{N}$ formed with the lattice operations in $C(X)$.

If $f \in C(X)$ is superinvariant, $f \geqq T^{*} f$, then $f$ is a potential with nonnegative charge $u=\left(1-T^{*}\right) f \geqq 0$. We denote by $\mathscr{K}$ the set of superinvariant functions in $C(X)$. Then $\mathscr{K}$ is a closed wedge in $C(X)$. It is well known that if $f, g \in \mathscr{K}$ then $f \wedge g \in \mathscr{K}$. Using the formula $\left(f_{1}-g_{1}\right) \wedge\left(f_{2}-g_{2}\right)=\left[\left(f_{1}+g_{2}\right) \wedge\left(f_{2}+g_{1}\right)\right]-\left(g_{1}+g_{2}\right)$, we see that the subspace $\mathscr{K}-\mathscr{K}$ of the potentials is a lattice. It is straightforward that $f \in \mathscr{K}-\mathscr{K}$ if and only if $\left|\left(1-T^{*}\right) f\right|$ is a charge such that $N\left|\left(1-T^{*}\right) f\right|$ is bounded. If we call $N\left|\left(1-T^{*}\right) f\right|$ the variation of $f$ then $\mathscr{K}-\mathscr{K}$ are the potentials of bounded variation. (An example below will justify the appellation.)

THEOREM 6. $\quad \mathscr{V}^{i v}=\{$ norm closure of $\mathscr{K}-\mathscr{K}$ in $C(X)\}$ is suitable, and is the smallest suitable of containing the superinvariant functions in $C(X)$.

Proof. Since $\mathscr{K}-\mathscr{K}$ is a lattice in $C(X)$ containing the constants, $\mathscr{Y}^{-i v}$ is a norm closed subalgebra of $C(X)$ containing the constants, by the Stone-Weierstrass theorem. If we can show that $\mathscr{K}-\mathscr{K}$ has properties (ii)-(iv) then straightforward approximation arguments will establish (ii)-(iv) for $\mathscr{V}^{-i v}$. First, if $f \in \mathscr{K}$ is superinvariant then $T^{*} f$ is superinvariant, whence $T^{*} f \in \mathscr{K}-\mathscr{K}$ if $f \in \mathscr{K}-\mathscr{K}$. Next, $\mathscr{K}=\mathscr{K} \cap(-\mathscr{K})$, whence $\mathscr{K} \subset \mathscr{K} \subset \mathscr{K}-\mathscr{K}$. Finally, if $f \in \mathscr{K}$ then $f \geqq T^{*} f \geqq \cdots \geqq Q(\lambda) f$, whence $f-Q(\lambda) f \geqq 0$. Since $Q(\lambda)[f-Q(\lambda) f]=0$, it must be the case that $f-Q(\lambda) f=0$ on $\mathscr{S}\left(q_{x}^{(\lambda)}\right)$ when $x \in \theta^{-1} B$. The same is true for $f \in \mathscr{K}-\mathscr{K}$. Thus $\mathscr{K}-\mathscr{K}$ and hence $\mathscr{V}^{-i v}$ has properties (ii)-(iv).

TheOREM 7. $\mathscr{V}^{\prime \prime \prime \prime}=\left\{f \in C(X):\right.$ for some $\lambda, w^{*}-\lim T^{* n}|f-Q(\lambda) f|=$ $0\}$ is suitable. If $f \in \mathscr{V}^{\prime \prime \prime \prime}$ then the defining relation is satisfied for every $\lambda$. A weak averaging characterization of $\mathscr{V}^{\prime \prime \prime}$ is given by $\mathscr{V}^{\prime \prime \prime \prime}=\left\{f \in C(X): w^{*}-\lim T^{* n}[g(f-Q(\lambda) f)]=0\right.$ for each $\left.g \in C(X)\right\}$.

Proof. We observe first that $\mathscr{V}^{\prime \prime \prime \prime}$ is a norm closed subspace of $C(X)$; a suitable pseudonorm is $p(f)=\left\|\operatorname{Lim} \operatorname{Sup} T^{* n}|f-Q(\lambda) f|\right\|$. Since $\left|T^{*} f-Q(\lambda) T^{*} f\right|=\left|T^{*}(f-Q(\lambda) f)\right| \leqq T^{*}|f-Q(\lambda) f|$, we have 
$T^{*} f \in \mathscr{Y}^{\prime \prime \prime \prime}$ if $f \in \mathscr{Y}^{\prime \prime \prime \prime}$, and condition (ii) is satisfied. It is obvious that condition (iii) is satisfied. If $f \in \mathscr{Y}^{\prime \prime \prime \prime}$ then for every $\lambda_{1}$,

$$
Q\left(\lambda_{1}\right)|f-Q(\lambda) f|=w^{*}-\lim T^{* n}|f-Q(\lambda) f|=0,
$$

whence $f-Q(\lambda) f=0$ on $\mathscr{S}\left(q_{x}^{\left(\lambda_{1}\right)}\right)$ for each $x \in \theta^{-1} B$ and each $\lambda_{1}$. Not only is condition (iv) satisfied, but $\mathscr{V}^{\prime \prime \prime} \subset \mathscr{Y}^{\prime \prime \prime}$ and the defining relation for $\mathscr{y}^{\prime \prime \prime \prime}$ is independent of $\lambda$. From $\left|T^{* n}(f-Q(\lambda) f)\right| \leqq T^{* n}|f-Q(\lambda) f|$ if follows that each $f \in \mathscr{V}^{\prime \prime \prime}$ is a potential. It remains to show that $\mathscr{Y}^{\prime \prime \prime \prime}$ is an algebra. First, we observe that if $f \in \mathscr{K}$ then $f-Q(\lambda) f \geqq 0$ and $T^{* n}(f-Q(\lambda) f) \downarrow 0$, so $\mathscr{K} \subset \mathscr{Y}^{\prime \prime \prime}$. Since $\mathscr{V}^{\prime \prime \prime}$ is closed, $\mathscr{V}^{-i v} \subset \mathscr{V}^{\prime \prime \prime}$. Suppose $f, g \in \mathscr{V}^{\prime \prime \prime \prime}$. Then

$$
\begin{aligned}
|f g-Q(\lambda)(f g)| & =\mid f(g-Q(\lambda) g)+(f-Q(\lambda) f) Q(\lambda) g \\
& +Q(\lambda) f \cdot Q(\lambda) g-Q(\lambda)(f g) \mid \\
\leqq & || f|||g-Q(\lambda) g|+|| g|||f-Q(\lambda) f| \\
& +|Q(\lambda) f \cdot Q(\lambda) g-Q(\lambda)(f g)| .
\end{aligned}
$$

Since $\mathscr{Y}^{\prime \prime \prime} \subset \mathscr{Y}^{\prime \prime}$ we have $Q(\lambda)(f g)=Q(\lambda)[Q(\lambda) f \cdot Q(\lambda) g]$, and the last term on the right hand side in the above inequality is of the form $|h-Q(\lambda) h|$ with $h=Q(\lambda) f \cdot Q(\lambda) g$. Now $Q(\lambda) f, Q(\lambda) g \in \mathscr{C}$, so we have $h \in \mathscr{Y}^{i v} \subset \mathscr{V}^{\prime \prime \prime \prime}$, and finally $f g \in \mathscr{V}^{\prime \prime \prime \prime}$. Thus condition (i) is satisfied. A weak averaging characterization of $\mathscr{V}^{\prime \prime \prime}$ is given by

$\mathscr{Y}^{\prime \prime \prime}=\left\{f \in C(X): w^{*}-\lim T^{* n}[g(f-Q(\lambda) f)]=0\right.$ for each $\left.g \in C(X)\right\}$.

To see this, we observe first that each $f \in \mathscr{V}^{\prime \prime \prime \prime ~ s a t i s f i e s ~ t h e ~ c o n d i t i o n ~}$ in braces. Suppose $f \in C(X)$ satisfies the condition. With

$$
g=f-Q(\lambda) f,
$$

we have

$$
w^{*}-\lim T^{* n}(f-Q(\lambda) f)^{2}=0,
$$

and since

$$
T^{* n}|f-Q(\lambda) f| \leqq\left[T^{* n}(f-Q(\lambda) f)^{2}\right]^{\frac{1}{2}},
$$

we obtain $f \in \mathscr{Y}^{\prime \prime \prime}$.

As we saw in $\S 3$, for each open closed subset $C$ of $B$ there is an open closed subset $C^{\vee}$ of $X$ such that $\chi_{C^{\vee}} \leqq T^{*} \chi_{C^{\vee}} \leqq \cdots \uparrow Q(\lambda) \chi_{C^{\vee}}=$ $\Phi \chi_{C}$. Since $-\chi_{C^{\vee}} \in \mathscr{K}$, each of $\mathscr{Y}^{\prime \prime}-\mathscr{V}^{i v}$ above contains each such $\chi_{C^{\vee}}$. In each of $Y^{\prime}-Y^{i v}$, the set $C_{1}=v C^{\vee}$ is an open closed subset of $Y$ such that $C=B \cap C_{1}$; moreover, $\chi_{c_{1}} \leqq S \chi_{C_{1}} \leqq \cdots \uparrow Q_{0}(\lambda) \chi_{C_{1}}$ for the topology induced by the special measures.

Finally, we let $\mathscr{Y}^{-v}$ be the intersection of all $\mathscr{Y}$ satisfying con- 
ditions (i)-(iv) for all $\lambda$. As we have shown, the set of such $\mathscr{V}$ is not empty, and $\mathscr{V}^{\sim v}$ exists. It may described as follows. Put $\mathscr{C}_{0}=\mathscr{C}$, define $\mathscr{l l}_{n}$ for odd $n$ as the linear span of all products $f g$ with $f$, $g \in \mathscr{C}_{n-1}$, and define $\mathscr{C}_{n}$ for even $n \geqq 2$ as $\mathscr{C}_{n}=\operatorname{span}\left(\mathscr{C}_{n-1}, T^{*} \mathscr{C}_{n-1}\right)$. If $f, g \in \bigcup_{n} \mathscr{C}_{n}$ then $f g \in \bigcup_{n} \mathscr{C}_{n}$ and $T^{*} f \in \bigcup_{n} \mathscr{C}_{n} ; \mathscr{V}^{* v}$ is the norm closure of $\bigcup_{n} \mathscr{C l}_{n}$. The author has not been able to obtain a more intrinsic description of $\mathscr{V}^{v v}$.

The following examples will illustrate the considerations involved above. Let $N=\{\cdots,-1,0,1, \cdots\}$ be the integers, and let $L$ be $l(N)$, the norm closed span of the point measures $\left\{\delta_{n}: n \in N\right\}$. Let $T$ be determined by $T \delta_{n}=\delta_{n+1}, n \in N$. Then $X$ is the Stone-Cech compactification $\beta N$ of $N$. Any Banach limit $\lambda$ may be regarded as a $T^{* *}$ invariant probability $\lambda^{\prime}$ on $\beta N$, with $\mathscr{S}\left(\lambda^{\prime}\right) \subset \beta N_{+}$where $N_{+}=\{1,2, \cdots\}$. The only $T^{*}$ invariant functions in $C(X)$ are the constants. The projection $Q(\lambda)$ has the form $Q(\lambda) f=\left(f, \lambda^{\prime}\right) \cdot 1$, whence $q_{x}^{(\lambda)}=\lambda^{\prime}$ for all $x \in X$. The Feller boundary consists of a single point, and $\theta^{-1} B=X$. It is known that $\bigcup_{2} \mathscr{S}\left(\lambda^{\prime}\right)$ is relatively nowhere dense in $\beta N_{+}-N_{+}$, as is each $\mathscr{S}\left(\lambda^{\prime}\right)$ [1]. If we fix $\lambda$ and collapse $\mathscr{S}\left(\lambda^{\prime}\right)$ to a single point $B$, we have $Y^{\prime}$, above. If we collapse $\left[\bigcup_{\lambda} \mathscr{S}\left(\lambda^{\prime}\right)\right]^{-}$to a point $B$ then we have $Y^{\prime \prime}$. Each member of $C\left(Y^{\prime \prime}\right)$ is almost convergent on $N_{+}$. (Not every almost convergent sequence appears in $C\left(Y^{\prime \prime}\right)$, however; e.g., $f(n)=(-1)^{n}$ extends to a function which is \pm 1 on $\beta N$ with $\left(f, \lambda^{\prime}\right)=0$. This function cannot be constant on $\mathscr{S}\left(\lambda^{\prime}\right)$.) It is clear that $\mathscr{Y}^{\prime \prime \prime}$ consists of the functions such that $\lim _{n \rightarrow+\infty} f(n)$ exists. Space $Y^{\prime \prime \prime}$ is $N$ compactified with $\beta N_{-}-N_{-}$at $-\infty$ and the one point $B$ at $+\infty$. The superinvariant functions on $\beta N$ are the functions monotone decreasing on $N, \mathscr{K}-\mathscr{K}$ consists of the functions of bounded variation on $N$, and $\mathscr{V}^{-i v}$ are the functions such that $\lim _{n \rightarrow+\infty} f(n)$ and $\lim _{n \rightarrow-\infty} f(n)$ exist, not necessarily equal. Space $Y^{i v}$ is the two point compactification of $N$, with $B$ the point at $+\infty$. The only functions in $\mathscr{Y}^{v v}$ are the constants, and $Y^{v}$ is the one point $B$. In $Y^{\prime}-Y^{i v}$, the special measures are just $l(N)$; in each instance, $C(Y)$ is not the conjugate space of the special measures.

For another example, let $L$ and $X$ be as above, but with $T \delta_{n}=$ $\frac{1}{2} \delta_{n-1}+\frac{1}{2} \delta_{n+1}, n \in N$. Again, the only invariant functions are the constants. The projection measure $q_{x}^{(\lambda)}$ is independent of $x \in X$ and is a Banach limit on $\beta N$ with half its support in $\beta N_{+}$and half in $\beta N_{-}$. In $Y^{\prime}$ and $Y^{\prime \prime}$, the two ends of $\beta N$ are stuck together along $\mathscr{S}\left(q_{x}^{(2)}\right)$ and $\left[\bigcup_{\lambda} \mathscr{S}\left(q_{x}^{(\lambda)}\right)\right]^{-}$, respectively. If $f \in C(X)$ is such that $\lim _{n \rightarrow \infty} f(n)=$ $\lim _{n \rightarrow-\infty} f(n)$ then $f \in \mathscr{V}^{\prime \prime \prime \prime}$, by the central limit theorem. However, $\mathscr{V}^{\prime \prime \prime \prime}$ is larger than this; e.g., if $f\left(2^{m}\right)=1$ for $m \in N_{+}$and $f(n)=0$ for the other $n \in N$ then $f \in \mathscr{Y}^{\prime \prime \prime}$, so that $Y^{\prime \prime \prime}$ is larger than the one point compactification of $N$. The superinvariant functions are the 
functions concave on $N$, and the only bounded ones are the constants. Thus $\mathscr{Y}^{i v}=\mathscr{Y}^{v v}=$ constants and $Y^{i v}=Y^{v}=\{B\}$.

REMARK. The nice boundary for this example is probably the two point compactification. It seems likely that to get it one must consider unbounded superinvariant functions, which do not fit our framework.

5. The separable case. We make the assumption now that the Banach space $L$ is separable. Under this assumption, $L$ will have an $F$ unit $\pi$, and $\kappa \pi$ will be a category measure on $X ; L$ is isomorphic to $L_{1}(X, \kappa \pi)$. The measure $\Phi^{*} \kappa \pi$ is a category measure for $B$, and the space of normal measures on $B$ is isomorphic to $L_{1}\left(B, \Phi^{*} \kappa \pi\right)$.

When $L$ is separable the quotient space $\mathscr{R}=L / \mathscr{N}$ of $\S 2$ is also separable, as is then the isomorphic $L_{1}\left(B, \Phi^{*} \kappa \pi\right)$.

It is well known that this implies that the weak* topology of the unit ball of $C(B)$ is metrizable. Let $E_{0}=B, E_{1}, \cdots$ be a countable collection of open closed subsets of $B$ such that $\left\{\chi_{E_{n}}\right\}$ is total on $L_{1}\left(B, \Phi^{*} \kappa \pi\right)$. Define $\xi: B \rightarrow B_{0}$ by

$$
\xi(z)=2 \sum_{1}^{\infty} \frac{\chi_{E_{n}}(z)}{3^{n}}, \quad z \in B,
$$

so that $B_{0}$ is a closed subset of the Cantor set. Let $\Xi: C\left(B_{0}\right) \rightarrow C(B)$ be the induced injection. For each $n$ there is an open closed subset $F_{n}$ of $B_{0}$ such that $\chi_{E_{n}}=\Xi \chi_{F_{n}}$, whence the range of $\Xi$ is the norm closed subalgebra of $C(B)$ generated by $\left\{\chi_{E_{n}}\right\}$. If ca $\left(B_{0}\right)$ are the set functions countably additive on $\left(B_{0}\right.$, Borel sets), then each member of ca $\left(B_{0}\right)$ is regular. The adjoint $\Xi^{*}:$ rca $(B) \rightarrow$ ca $\left(B_{0}\right)$ sends $\Phi^{*} \kappa \pi$ to a measure $\pi_{0}=\Xi^{*} \Phi^{*} \kappa \pi$ in ca $\left(B_{0}\right)$ with $\mathscr{S}\left(\pi_{0}\right)=B_{0}$. As we have noted before, the mapping $\Xi^{*} L_{1}\left(B, \Phi^{*} \kappa \pi\right) \rightarrow L_{1}\left(B_{0}, \pi_{0}\right)$ is onto. Suppose $\nu_{1}$ and $\nu_{2}$ are distinct members of $L_{1}\left(B, \Phi^{*} \kappa \pi\right)$. Then $\nu_{1}\left(E_{n}\right) \neq \nu_{2}\left(E_{n}\right)$ for at least one $n,\left\{\chi_{E_{n}}\right\}$ being total. Since $\nu\left(E_{n}\right)=\left(\Xi^{*} \nu\right)\left(F_{n}\right)$ with $F_{n}$ as above, we have $\left(\Xi^{*} \nu_{1}\right)\left(F_{n}\right) \neq\left(\Xi^{*} \nu_{2}\right)\left(F_{n}\right)$ for at least one $n$, whence $\Xi^{*} \nu_{1}$ and $\Xi^{*} \nu_{2}$ are distinct members of $L_{1}\left(B_{0}, \pi_{0}\right)$. This is to say, the mapping $\Xi^{*} L_{1}\left(B, \Phi^{*} \kappa \pi\right) \rightarrow L_{1}\left(B_{0}, \pi_{0}\right)$ is an isomorphism; it is clearly isometric and order preserving. It follows that $L_{\infty}\left(B, \Phi^{*} \kappa \pi\right)$ is isomorphic to $L_{\infty}\left(B_{0}, \pi_{0}\right)$. But $B$ is hyperstonian with $\Phi^{*} \kappa \pi$ as category measure, and it is known that $L_{\infty}\left(B, \Phi^{*} \kappa \pi\right)$ is isomorphic to $C(B)$. We have proved

Theorem 8. Suppose $L$ is separable, with $\pi$ as $F$ unit. Then there is a closed subset $B_{0}$ of the Cantor set and a measure $\pi_{0}$ with $\mathscr{S}\left(\pi_{0}\right)=B_{0}$ such that

(i) the Feller boundary $B$ is the Boolean space of the measure 
algebra $\left(B_{0}\right.$, Borel sets, $\left.\pi_{0}\right)$,

(ii) $C(B)$ and hence $\mathscr{C l}$ is isometrically algebraically and lattice isomorphic to $L_{\infty}\left(B_{0}, \pi_{0}\right)$

(iii) the space $L_{1}\left(B, \Phi^{*} \kappa \pi\right)$ of normal measures on $B$ is isomorphic to $L_{1}\left(B_{0}, \pi_{0}\right)$.

This result is close to that of Feldman [4] concerning the Martin boundary of a countable state space Markov process.

An extension of our arguments will give a more detailed model. We consider one of the quotient spaces $Y$ discussed in $\S 4$. We will require that the elements of $\mathscr{V}^{-}$be potentials, so $\mathscr{Y}^{-}$will be one of $\mathscr{V}^{\prime \prime \prime}-\mathscr{V}^{-v}$. The mapping $\Theta^{*} L_{1}(X, \kappa \pi) \rightarrow L_{1}\left(Y, \Theta^{*} \kappa \pi\right)$ is onto the set of special measures, and $L_{1}\left(Y, \Theta^{*} \kappa \pi\right)$ is separable. The topology in $C(Y)$ induced by $L_{1}\left(Y, \Theta^{*} \kappa \pi\right)$ is just the relative weak topology of $\mathscr{V} \subset C(X)$, and the unit ball is metrizable. Let $\left\{f_{j}^{\prime}\right\}$ be a countable set in $C(Y)$ which is total on $L_{1}\left(Y, \Theta^{*} \kappa \pi\right)$. We have

$$
f_{j}^{\prime}=\left(f_{j}^{\prime}-Q_{0} f_{j}^{\prime}\right)+Q_{0} f_{j}^{\prime}
$$

with $f_{j}^{\prime}-Q_{0} f_{j}^{\prime}=0$ on $B \subset Y$ and $Q_{0} f_{j}^{\prime}$ invariant. We replace $f_{j}^{\prime}$ by $f_{j}=f_{j}^{\prime}-Q_{0} f_{j}^{\prime}$ and we replace the invariant parts by a countable system $\left\{g_{k}\right\}$ such that (i) $\Theta g_{k}=\Phi \chi_{E_{k}}$ for some open closed subset $E_{k}$ of $B$, (ii) $\left\{f_{j}\right\} \cup\left\{g_{k}\right\}$ is total on $L_{1}\left(Y, \Theta^{*} \kappa \pi\right)$, (iii) $\left\{\chi_{E_{k}}\right\}$ is total on $L_{1}\left(B, \Phi^{*} \kappa \pi\right)$. We normalize so that $\left\|f_{j}\right\|=1$.

Let $I^{\omega}$ be the Cartesian product of a countable collection of intervals $[-1,1]$. We metrize $I^{\omega}$ with the familiar

$$
d\left(z^{\prime}, z^{\prime \prime}\right)=\sum_{i=1}^{\infty} \frac{\left|z_{i}^{\prime}-z_{i}^{\prime \prime}\right|}{2^{i}}, \quad z^{\prime}, z^{\prime \prime} \in I^{\omega} .
$$

Define $\eta: Y \rightarrow K$ by

$$
\eta(y)=\left(f_{1}(y), g_{1}(y), f_{2}(y), g_{2}(y), \cdots\right), \quad y \in Y ;
$$

the range $K$ of $\eta$ is a closed subset of $I^{\omega}$. Let $B_{1}$ be the subset $\{\eta(y): y \in B\}$ of $K$. For $y \in B \subset Y$ we have

$$
\eta(y)=\left(0, \chi_{\Gamma_{1}}(y), 0, \chi_{E_{2}}(y), \cdots\right), \quad y \in B,
$$

and if we define

$$
\xi_{1}(z)=2 \sum_{1}^{\infty} \frac{g_{k}}{3^{k}}
$$

if

$$
z=\left(f_{1}, g_{1}, f_{2}, g_{2}, \cdots\right) \in I^{\omega}
$$

then the restriction of $\xi_{1}$ to $B_{1} \subset K$ is a homeomorphism of $B_{1}$ onto the subset $B_{0}$ of the Cantor set described at the beginning of the 
present section. (We assume that the families $\left\{E_{k}\right\}$ are the same, of course.)

The induced injection $H: C(K) \rightarrow C(Y)$ has as range the closed subalgebra of $C(Y)$ generated by the coordinate functions $\left\{f_{j}\right) \cup\left\{g_{k}\right\}$. The adjoint $H^{*}$ : rca $(Y) \rightarrow$ ca $(K)$ sends $\Theta^{*} \kappa \pi$ to $\rho=H^{*} \Theta^{*} \kappa \pi$, and $\mathscr{S}(\rho)=K$. The measure $Q_{0}^{* *} \Theta^{*} \kappa \pi$ on $Y$, which is the relative category measure $\Phi^{*} \kappa \pi$ for $B \subset Y$, becomes $\pi_{1}=H^{*} Q_{0}^{*} \Theta \kappa \pi \in$ ca $(K)$ with $\mathscr{S}\left(\pi_{1}\right)=B_{1} \subset K$, and $L_{1}\left(B_{0}, \pi_{0}\right)$ is isomorphic to $L_{1}\left(B_{1}, \pi_{1}\right)$.

It will be convenient to use $\tau^{\prime}=\Theta^{*} \kappa \pi+Q_{0}^{*} \Theta^{*} \kappa \pi$ as the reference measure in $Y$, so that $H^{*} \tau^{\prime}=\rho^{\prime}=\rho+\pi_{1}$ is the reference measure in $K$. The mapping $H^{*} L_{1}\left(Y, \tau^{\prime}\right) \rightarrow L_{1}\left(K, \rho^{\prime}\right)$ is onto. If $\nu_{1}$ and $\nu_{2}$ are distinct members of $L_{1}\left(Y, \tau^{\prime}\right)$ then $\left(h, \nu_{1}\right) \neq\left(h, \nu_{2}\right)$ for at least one $h \in\left\{f_{j}\right\} \cup\left\{g_{k}\right\}$. But these are just the coordinates in $K$; we have $H^{*} \nu_{1} \neq H^{*} \nu_{2}$ and the restriction $H^{*} L_{1}\left(Y, \tau^{\prime}\right) \rightarrow L_{1}\left(K, \rho^{\prime}\right)$ is an isomorphism. Let $J: L_{1}\left(K, \rho^{\prime}\right) \rightarrow L_{1}\left(Y, \tau^{\prime}\right)$ be the inverse, so that $H^{*} J=1$ and $J H^{*} \nu=\nu$ for $\nu \in L_{1}\left(Y, \tau^{\prime}\right)$. It should be obvious that $J L_{1}(K, \rho) \rightarrow$ $L_{1}\left(Y, \Theta^{*} \kappa \pi\right)$ and $J L_{1}\left(K, \pi_{1}\right) \rightarrow L_{1}\left(Y, Q_{0}^{*} \Theta^{*} k \pi\right)$ are subisomorphisms. The adjoint $J^{*}: L_{\infty}\left(Y, \tau^{\prime}\right) \rightarrow L_{\infty}\left(K, \rho^{\prime}\right)$ does several things. First, it is a straightforward matter to show that $L_{\infty}\left(Y, \tau^{\prime}\right)$ is isomorphic to the weak* closure of $\mathscr{V}^{*}$ in $C(X) ; J^{*}$ is in effect an isomorphism of this closure onto $L_{\infty}\left(K, \rho^{\prime}\right)$. This may be described as follows. If $f \in L_{\infty}\left(Y, \tau^{\prime}\right)$ then there is a sequence $\left\{u_{n}\right\}$ in the linear span of $\left\{f_{j}\right\} \cup\left\{g_{k}\right\}$ such that $w^{*}-\lim u_{n}=f$. Each $u_{n}$ is the image $u_{n}=H v_{n}$ of $v_{n} \in C(K)$, and $J^{*} f=w^{*}-\lim v_{n}$. We extend the definition of $H$ to $L_{\infty}\left(K, \rho^{\prime}\right)$ by $H J^{*} f=f$, and have $J^{*} H=1$. Next, the restriction of $J^{*}$ to $C(Y)$ produces an isomorphism of $C(Y)$ onto a subalgebra of $L_{\infty}\left(K, \rho^{\prime}\right)$ which we denote by $\mathscr{B}$. Finally, the restriction of $J^{*}$ to $H C(K)$ is an isomorphism of $H C(K)$ onto $C(K)$.

The transition operator $S^{*}$ on rca $(Y)$ sends the special measures $L_{1}\left(Y, \Theta^{*} \kappa \pi\right)$ into special measures and is the identity on $L_{1}\left(Y, Q_{0}^{*} \Theta^{*} \kappa \pi\right)$, from which it is easy to see that $S^{*}$ sends $L_{1}\left(Y, \tau^{\prime}\right)$ into $L_{1}\left(Y, \tau^{\prime}\right)$. By virtue of the isomorphism $J$ we obtain a transition operator $U: L_{1}\left(K, \rho^{\prime}\right) \rightarrow L_{1}\left(K, \rho^{\prime}\right)$ according to $U=H^{*} S^{*} J$. Since $K$ is closed in $I^{\omega}$, the kernel of $U$ may be determined as a regular conditional probability, and a Markov process with $K$ as state space can be defined in the usual way. Only the probabilities in $L_{1}(K, \rho)$ will be considered as initial propabilities. The process in $K$ is not necessarily a Feller process.

If $f \in C(Y)$ is invariant, $f=S f$, then $J^{*} f \in \mathscr{B}$ will be invariant for $U^{*}: L_{\infty}\left(K, \rho^{\prime}\right) \rightarrow L_{\infty}\left(K, \rho^{\prime}\right)$. Conversely, if $g \in L_{\infty}\left(K, \rho^{\prime}\right)$ is invariant then $H g \in L_{\infty}\left(Y, \tau^{\prime}\right)$ will be invariant for the extension of $S$ to $L_{\infty}\left(Y, \tau^{\prime}\right)$ (viz., the restriction of $T^{*}$ to the weak* closure of $\mathscr{V}^{*}$.) Since every invariant function in $C(X)$ is in $\mathscr{y}^{\prime}$, we must have $H g \in C(Y)$. This is to say, every $U^{*}$ invariant function in $L_{\infty}\left(K, \rho^{\prime}\right)$ is in fact an ele- 
ment of $\mathscr{B}$. The restriction of these invariant functions to $B_{1} \subset K$ gives an isomorphism onto $L_{\infty}\left(B_{1}, \pi_{1}\right)$, in turn isomorphic to $C(B)$ and $\mathscr{l l}$. The projection $Q_{0}$ in $C(Y)$ gives rise to a projection $Q_{1}: L_{\infty}\left(K, \rho^{\prime}\right) \rightarrow$ $L_{\infty}\left(K, \rho^{\prime}\right)$ onto the $U^{*}$ invariant functions, according to $Q_{1}=J^{*} Q_{0}^{\prime} H$. Here, $Q_{0}^{\prime}$ is the extension of $Q_{0}$ to $L_{\infty}\left(Y, \tau^{\prime}\right)$ (viz., the restriction of $Q(\lambda)$ to the weak* closure of $\mathscr{V}^{*}$.) It is easily verified that $Q_{1}^{*}$ sends $L_{1}\left(K, \rho^{\prime}\right)$ into $L_{1}\left(K, \rho^{\prime}\right)$, implying that $Q_{1}$ is an adjoint. Using this and the fact that $B_{1} \subset K$ is homeomorphic to a closed subset of the Cantor set, we may represent the action of $Q_{1}$ by a regular kernel:

$$
\left(Q_{1} f\right)(x)=\int f\left(x^{\prime}\right) q_{1}\left(x, d x^{\prime}\right), \quad x \in K, f \in L_{\infty}\left(K, \rho^{\prime}\right),
$$

where $q_{1}(x, E)$ is a countably additive probability in Borel $E \subset K$ for each $x \in K$ and a Borel function of $x \in K$ for each fixed Borel $E \subset K$. For each $x \in K$ we have $\mathscr{S}\left(q_{1}(x, \cdot)\right) \subset B_{1}$, of course.

We examine now our assumption that each $\theta f, f \in C(Y)$ is a potential. We have

$$
\left(S^{n} f, \nu\right) \longrightarrow\left(Q_{0} f, \nu\right)
$$

for each special measure $\nu \in L_{1}\left(Y_{1}, \Theta^{*} \kappa \pi\right)$ and each $f \in C(Y)$, so that

$$
\left(f, S^{* n} \nu\right) \longrightarrow\left(f, Q_{0}^{*} \nu\right), f \in C(Y), \nu \in L_{1}\left(Y, \Theta^{*} \kappa \pi\right) \text {. }
$$

Dropping to $K$, we obtain

$$
\left(g, H^{*} S^{* n} J \mu\right) \longrightarrow\left(g, H^{*} Q_{0}^{*} J \mu\right), g \in C(K), \mu \in L_{1}(K, \rho) .
$$

If we use the fact that $S^{*}$ operates on $L_{1}\left(Y, \tau^{\prime}\right)$ we obtain $H^{*} S^{* n} J=$ $U^{n}$, so we have

$$
\left(g, U^{n} \mu\right) \longrightarrow\left(g, H^{*} Q_{0}^{*} J \mu\right), g \in C(K), \mu \in L_{1}(K, \rho) .
$$

This is to be interpreted as follows. Let $\left\{z_{0}(\omega), z_{1}(\omega), \cdots\right\}$ be the random variables of the Markov process with $K$ as state space, $\mu \in L_{1}(K, \rho)$ as initial probability, and $U$ as transition operator. Then $U^{n} \mu$ is the marginal distribution of $z_{n}(\omega)$. The relation above is the assertion that this sequence of marginal distributions converges weakly* in ca $(K)$ to the measure $H^{*} Q_{0}^{*} J \mu \in$ ca $(K)$. This measure will have support in $B_{1} \subset K$, and will be an element of $L_{1}\left(B, \pi_{1}\right)$, but not necessarily of $L_{1}(K, \rho)$.

Now we get down to cases. For $Y$ above we take the space $Y^{i v}$ of $\S 4$. We choose the separating family $\left\{f_{j}^{\prime}\right\}$ so that each $f_{j}^{\prime} \in \mathscr{K}$. Then each member of $\left\{f_{j}\right\} \cup\left\{g_{k}\right\}$ is superinvariant, $h \geqq S h$ for all $h \in\left\{f_{j}\right\} \cup\left\{g_{k}\right\}$. It is straightforward that if $\left\{z_{0}(\omega), z_{1}(\omega), \cdots\right\}$ is the Markov process in $K$ discussed above, then for each fixed $i$ the $i$-th 
coordinate process $\left[z_{0}(\omega)\right]_{i},\left[z_{1}(\omega)\right]_{i}, \cdots$ is a supermartingale relative to the increasing $\sigma$-fields of the Markov process. We invoke the supermartingale convergence theorem: $z_{n}(\omega)$ converges with probability 1 to a random variable $z(\omega) \in K$. (It is essential to the argument that the number of coordinates is countable, of course.) The discussion of the preceding paragraph shows that the limit point $z(\omega)$ has $H^{*} Q_{0}^{*} J \mu$ as distribution, and lies in the boundary $B_{1}$ with probability 1 . Thus we have obtained a model in which the process converges to a point of the boundary with probability 1 .

Instead of $Y^{i v}$ let us use $Y^{\prime \prime \prime}$. We have $\left(S^{n}\left|f_{j}\right|, \nu\right) \rightarrow 0$ for each $\nu \in L_{1}\left(Y_{1}\left(Y, \Theta^{*} \kappa \pi\right)\right.$. In the process $\left\{z\left(\omega_{0}\right), z_{1}(\omega), \cdots\right\}$, the coordinate processes $\left[z_{0}(\omega)\right]_{i},\left[z_{1}(\omega)\right]_{i}, \cdots$ for even values of $i$ are martingales, and for odd values of $i$ converge in probability to 0 . Thus $p-\lim z_{n}(\omega)=$ $z(\omega)$ exists with probability 1 ; that is, $\lim _{n} \operatorname{Prob}\left\{d\left(z_{n}(\omega), z(\omega)\right) \geqq \varepsilon\right\}=0$ for each $\varepsilon>0$. Moreover, the distribution of the limiting random variable $z(\omega)$ is $H^{*} Q_{0}^{*} J \mu \in L_{1}\left(B_{1}, \pi_{1}\right)$, and the limiting point $z(\omega)$ lies in the boundary $B_{1}$ with probability 1 .

The examples of $\S 4$ indicate the difference between the two models. On a recurrence class the only superinvariant functions are the constants, and in $K^{i v}$ each recurrence class in $X$ is collapsed to a point of the boundary $B_{1}^{i v}$. A random point in a recurrence class is already in $B_{1}^{i v}$, and convergence to the boundary is trivial. The structure of the transient classes is preserved in $K^{i v}$, however, and the convergence to a boundary point with probability 1 is meaningful. In $K^{\prime \prime \prime}$ the recurrence classes need not be trivial, depending on the size of $\mathscr{V}^{-\prime \prime \prime}$. A random point in a recurrence class is converging in probability to a boundary point. It cannot converge with probability 1 , of course, since it must keep moving to cover the recurrence class. Only the null recurrent classes will be represented in a nontrivial way.

6. The general state space. We describe now the problem which motivated the present investigation, and sketch the extent to which the preceding results are applicable. Let $\left(X_{0}, \mathscr{F}\right)$ be a measurable space, and let $P(x, E)$ be a transition probability for $\left(X_{0}, \mathscr{F}\right)$. That is, $P(x, E)$ is a countably additive probability measure in $E \in \mathscr{F}$ for each fixed $x \in X_{0}$, and is an $\mathscr{F}$ measurable function of $x$ for each $E \in \mathscr{F}$. For the space $L$ we take the space ca $\left(X_{0}, \mathscr{F}\right)$ of countably additive set functions on $\mathscr{F}$, and we let $T$ be

$$
(T \mu)(E)=\int P(x, E) \mu(d x), \quad E \in \mathscr{F}, \mu \in \mathrm{ca}\left(X_{0}, \mathscr{F}\right) .
$$

The space $X$ is quite large and unwieldy, and its connection with $X_{0}$ would appear to be somewhat remote. We may simplify as follows, however. Let $\mu \in \mathrm{ca}\left(X_{0}, \mathscr{F}\right)$ be any initial probability. If we define 
$\mu^{\prime}=\sum_{0}^{\infty} 2^{-n-1} T^{n} \mu$, then $\mu \in L_{1}\left(X_{0}, \mathscr{F}, \mu^{\prime}\right)$ and

$$
T L_{1}\left(X_{0}, \mathscr{F}, \mu^{\prime}\right) \subset L_{1}\left(X_{0}, \mathscr{F}, \mu^{\prime}\right) \text {. }
$$

If $X_{1}$ is $\mathscr{S}\left(\kappa \mu^{\prime}\right)$ in $X$ then $L_{\infty}\left(X_{0}, \mathscr{F}, \mu^{\prime}\right)$ is isomorphic to $C\left(X_{1}\right)$ so that $X_{1}$ is just the Boolean space of the measure algebra $\left(X_{0}, \mathscr{F}, \mu^{\prime}\right)$. The intersection $\theta^{-1} B \cap \mathscr{S}\left(\kappa \mu^{\prime}\right)$ is the Choquet boundary of the invariant functions in $L_{\infty}\left(X_{0}, \mathscr{F}, \mu^{\prime}\right)$, and $\mathscr{S}\left(\Phi^{*} \kappa \mu^{\prime}\right)$ is the Feller boundary for the action of $T$ on $L_{1}\left(X_{0}, \mathscr{F}, \mu^{\prime}\right)$. Thus the big Feller boundary $B$ contains the Feller boundary for each initial probability $\mu$; each such piece has a category measure.

The various quotient structures associated with $X$ have substructures associated with each initial probability $\mu$. If $\mathscr{F}$ is countably generated then each $L_{1}\left(X_{0}, \mathscr{F}, \mu^{\prime}\right)$ will be separable, and $\S 5$ applies. We now show how the quotient boundaries can be related to $X_{0}$ in a more direct fashion.

Let $B\left(X_{0}, \mathscr{F}\right)$ be the algebra of $\mathscr{F}$ measurable functions on $X_{0}$, with pointwise operations and supremum norm. We define

$$
P: B\left(X_{0}, \mathscr{F}\right) \longrightarrow B\left(X_{0}, \mathscr{F}\right)
$$

by

$$
P f(x)=\int f\left(x^{\prime}\right) P\left(x, d x^{\prime}\right), \quad x \in X_{0}, f \in B\left(X_{0}, \mathscr{F}\right) .
$$

The Banach space conjugate of $B\left(X_{0}, \mathscr{F}\right)$ is the space ba $\left(X_{0}, \mathscr{F}\right)$ of finitely additive set functions on $\mathscr{F}$, and $T$ above is the restriction of the adjoint $P^{*}$ to the subspace ca $\left(X_{0}, \mathscr{F}\right)$ of ba $\left(X_{0}, \mathscr{F}\right)$.

Let $\mathscr{K}_{0}=\left\{f \in\left(B\left(X_{0}, \mathscr{F}\right): f \geqq P f\right\}\right.$ be the superinvariant functions, let $\mathscr{U}_{0}$ be the norm closure of the lattice $\mathscr{K}_{0}-\mathscr{K}_{0}$ in $B\left(X_{0}, \mathscr{F}\right)$, and let $\mathscr{G} \subset \mathscr{F}$ be the smallest $\sigma$-field with respect to which every function in $\mathscr{K}_{0}\left(\right.$ or $\left.\mathscr{U}_{0}\right)$ is measurable. With the initial probability $\mu$ chosen and fixed, the action of $T$ on $L_{1}\left(X_{0}, \mathscr{Y}, \mu^{\prime}\right)$ gives a transition operator for $\left(X_{0}, \mathscr{C}\right)$ and every invariant function in $L_{\infty}\left(X_{0}, \mathscr{F}, \mu^{\prime}\right)$ is in $L_{\infty}\left(X_{0}, \mathscr{G}, \mu^{\prime}\right)$. If $f \in \mathscr{K}_{0}$ then for each real $c$ we have

$$
\chi_{\{f \geqq c\}}=\lim _{\varepsilon \downarrow 0} \frac{f \wedge c-f \wedge(c-\varepsilon)}{\varepsilon}
$$

pointwise and boundedly, from which one sees that $\mathscr{K}_{0}$ is total on $L_{1}\left(X_{0}, \mathscr{G}, \mu^{\prime}\right)$, whence $\mathscr{L}_{0}$ is weakly* dense in $L_{\infty}\left(X_{0}, \mathscr{G}, \mu^{\prime}\right)$.

Let $\left\{h_{i}\right\}$ be a countable set in $\mathscr{K}_{0}$ chosen according to the recipe of $\S 5$ for $\left\{f_{j}\right\} \cup\left\{g_{k}\right\}$. Let $d$ be the pseudometric on $X_{0}$ defined by

$$
d\left(x^{\prime}, x^{\prime \prime}\right)=\sum_{1}^{\infty} 2^{-i}\left|h_{i}\left(x^{\prime}\right)-h_{i}\left(x^{\prime \prime}\right)\right|, \quad x^{\prime}, x^{\prime \prime} \in X_{0} .
$$

Each $h_{i}$ is continuous on $X_{0}$, and $\left\{h_{i}\right\}$ is total on $L_{1}\left(X_{0}, \mathscr{G}, \mu^{\prime}\right)$. We 
let $K$ denote the completion of $X_{0}$ with respect to $d$ (more precisely, the completion of $X_{0}$ after points at distance 0 are identified). It is straightforward that this $K$ is homeomorphic to $K^{i v}$ of $\S 5$, the basic space $L$ being $L_{1}\left(X_{0}, \mathscr{G}, \mu^{\prime}\right)$. There is a closed boundary set $B_{1} \subset K$ and a measure $\pi_{1}$ with $B_{1}$ as closed support such that the set of invariant functions in $L_{\infty}\left(X_{0}, \mathscr{G}, \mu^{\prime}\right)$, isomorphic to the invariant functions in $L_{\infty}\left(X_{0}, \mathscr{F}, \mu^{\prime}\right)$, is isomorphic to the whole of $L_{\infty}\left(B_{1}, \pi_{1}\right)$.

Let $\left\{x_{0}(\omega), x_{1}(\omega), \cdots\right\}$ be the random variables of the process with $\left(X_{0}, \mathscr{G}\right)$ as state space and any $\nu \in L_{1}\left(X_{0}, \mathscr{G}, \mu^{\prime}\right)$ as initial probability. Then $\lim x_{n}(\omega)=x(\omega) \in K$ exists with probability 1 , the limit $x(\omega)$ lies in the boundary $B_{1} \subset K$ with probability 1 , and the marginal distributions $T^{n} \nu$ of $x_{n}(\omega)$ tend to the distribution $\nu^{\prime}$ of $x(\omega)$ in the sense that $\left(T^{n} \nu, f\right) \rightarrow\left(\nu^{\prime}, f\right)$ for each $f \in C(K)$. The kernel of the projection $Q_{1}$ serves as a Poisson kernel for the invariant functions. That is, for each invariant function $f$ in $L_{\infty}\left(X_{0}, \mathscr{C}, \mu^{\prime}\right)$ we have

$$
f(x)=\int_{B_{1}} f_{1}\left(x^{\prime}\right) q_{1}\left(x, d x^{\prime}\right), \quad x \in X_{0},
$$

with $f_{1} \in L_{\infty}\left(K, \rho^{\prime}\right)$ being the invariant function in $L_{\infty}\left(K, \rho^{\prime}\right)$ uniquely determined by $f$.

If we use $\mathscr{V}^{\prime \prime \prime \prime}$ instead of $\mathscr{V}^{-i v}$ in the construction we obtain a completion in which $x_{n}(\omega)$ converges to a point of the boundary in probability instead of with probability 1 .

We have thus obtained several features of the Martin boundary [7]. There remains the problem of a kernel representation for superinvariant functions, and other considerations of potential theory. Further progress along these lines will be presented elsewhere.

\section{REFERENCES}

1. J. E. M. Alvarez de Araya, Invariant measures on compactification on the integers,

U. of Washington, 1963 Thesis (University Microfilms, Inc., Ann Arbor).

2. M. M. Day, Amenable semigroups, Illinois J. Math. 1 (1957), 509-544.

3. __ _ Normed Linear Spaces, Academic Press, Inc., New York, 1962.

4. Jacob Feldman, Feller and Martin boundaries for countable sets, Illinois J. Math. 6 (1962), 357-366.

5. W. Feller, Boundaries induced by nonnegative matrices, Trans. Amer. Math. Soc. 83 (1956), 19-54.

6. P. R. Halmos, On a theorem of Dieudonné, Proc. Nat. Acad. Sci. U.S.A. 35 (1949), $38-42$.

7. J. G. Kemeny, J. L. Snell, and A. W. Knapp, Denumerable Markov Chains, D. Van Nostrand Company, Inc., Princeton, 1966.

8. David G. Kendall, Hyperstonian spaces associated with Markov chains, Proc. London Math. Soc. (3) 10 (1960), 67-87.

9. S. P. Lloyd, On certain projections in spaces of continuous functions, Pacific J. J. Math. 13 (1963), 171-175.

10.

A mixing condition for extreme left invariant means, Trans. Amer. Math. 
Soc. 125 (1966), 461-481.

11. Paul A. Meyer, Probability and Potentials, Blaisdell Publishing Company, Waltham, 1966.

12. I. Namioka, On certain actions of semigroups on $L$ spaces (to appear)

13. Robert R. Phelps, Lectures on Choquet's Theorem, D. Van Nostrand Company, Inc., Princeton, 1966.

14. L. Sucheston, On existence of finite invariant measures, Math. Zietschr. 86 (1964), $327-336$.

Received November, 17, 1967.

Bell Telephone laboratories, Inc.

Murray Hill, New Jersey 


\section{PACIFIC JOURNAL OF MATHEMATICS}

\section{EDITORS}

\section{H. ROYDEN}

Stanford University

Stanford, California

\section{J. Dugundu}

Department of Mathematics University of Southern California Los Angeles, California 90007

RICHARD ARENS

University of California Los Angeles, California 90024

ASSOCIATE EDITORS
E. F. Beckenbach
B. H. NEUMANN
F. WoLF
K. YosidA

\section{SUPPORTING INSTITUTIONS}

\author{
UNIVERSITY OF BRITISH COLUMBIA \\ CALIFORNIA INSTITUTE OF TECHNOLOGY \\ UNIVERSITY OF CALIFORNIA \\ MONTANA STATE UNIVERSITY \\ UNIVERSITY OF NEVADA \\ NEW MEXICO STATE UNIVERSITY \\ OREGON STATE UNIVERSITY \\ UNIVERSITY OF OREGON \\ OSAKA UNIVERSITY \\ UNIVERSITY OF SOUTHERN CALIFORNIA
}

STANFORD UNIVERSITY

UNIVERSITY OF TOKYO

UNIVERSITY OF UTAH

WASHINGTON STATE UNIVERSITY

UNIVERSITY OF WASHINGTON

AMERICAN MATHEMATICAL SOCIETY
CHEVRON RESEARCH CORPORATION
TRW SYSTEMS

AMERICAN MATHEMATICAL SOCIETY

TRW SYSTEMS

NAVAL WEAPONS CENTER

Mathematical papers intended for publication in the Pacific Journal of Mathematics should be in typed form or offset-reproduced, double spaced with large margins. Underline Greek letters in red, German in green, and script in blue. The first paragraph or two must be capable of being used separately as a synopsis of the entire paper. It should not contain references to the bibliography. Manuscripts, in duplicate if possible, may be sent to any one of the four editors. All other communications to the editors should be addressed to the managing editor, Richard Arens, University of California, Los Angeles, California 90024.

Each author of each article receives 50 reprints free of charge; additional copies may be obtained at cost in multiples of 50 .

The Pacific Journal of Mathematics is published monthly. Effective with Volume 16 the price per volume (3 numbers) is $\$ 8.00$; single issues, $\$ 3.00$. Special price for current issues to individual faculty members of supporting institutions and to individual members of the American Mathematical Society: $\$ 4.00$ per volume; single issues $\$ 1.50$. Back numbers are available.

Subscriptions, orders for back numbers, and changes of address should be sent to Pacific Journal of Mathematics, 103 Highland Boulevard, Berkeley 8, California.

Printed at Kokusai Bunken Insatsusha (International Academic Printing Co., Ltd.), 7-17, Fujimi 2-chome, Chiyoda-ku, Tokyo, Japan.

PUBLISHED BY PACIFIC JOURNAL OF MATHEMATICS, A NON-PROFIT CORPORATION

The Supporting Institutions listed above contribute to the cost of publication of this Journal, but they are not owners of publishers and have no responsibility for its content or policies. 


\section{Pacific Journal of Mathematics \\ Vol. 27, No. 3}

March, 1968

Charles A. Akemann, Invariant subspaces of $C(G) \ldots \ldots \ldots \ldots \ldots \ldots . \ldots 41$

Dan Amir and Zvi Ziegler, Generalized convexity cones and their duals ... . 425

Raymond Balbes, On ( $J, M, \mathrm{~m})$-extensions of order sums of distributive

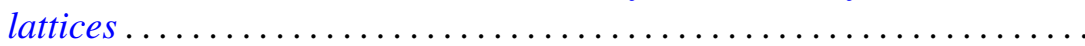

Jan-Erik Björk, Extensions of the maximal ideal space of a function algebra ........................................ 453

Frank Castagna, Sums of automorphisms of a primary abelian group ...... 463

Theodore Seio Chihara, On determinate Hamburger moment problems ..... .

Zeev Ditzian, Convolution transforms whose inversion function has complex

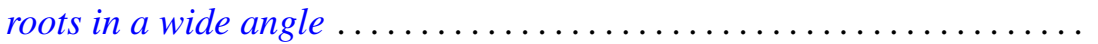

Myron Goldstein, On a paper of Rao .

Velmer B. Headley and Charles Andrew Swanson, Oscillation criteria for

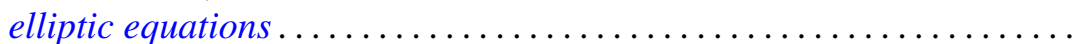

John Willard Heidel, Qualitative behavior of solutions of a third order nonlinear differential equation............................

Alan Carleton Hindmarsh, Pick's conditions and analyticity.............

Bruce Ansgar Jensen and Donald Wright Miller, Commutative semigroups

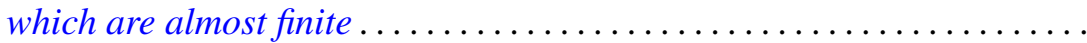

Lynn Clifford Kurtz and Don Harrell Tucker, An extended form of the

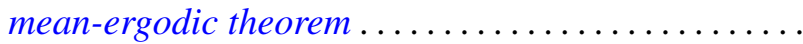

S. P. Lloyd, Feller boundary induced by a transition operator ...

Henry B. Mann, Josephine Mitchell and Lowell Schoenfeld, A new proof of the maximum principle for doubly-harmonic functions ...

Robert Einsohn Mosher, The product formula for the third obstruction ..

Sam Bernard Nadler, Jr., Sequences of contractions and fixed points ...

Eric Albert Nordgren, Invariant subspaces of a direct sum of weighted shifts...

Fred Richman, Thin abelian p-groups ...

Jordan Tobias Rosenbaum, Simultaneous interpolation in $\mathrm{H}_{2}$. II ...

Charles Thomas Scarborough, Minimal Urysohn spaces .

Malcolm Jay Sherman, Disjoint invariant subspaces..... .

Joel John Westman, Harmonic analysis on groupoids....

621

William Jennings Wickless, Quasi-isomorphism and TFM

Minoru Hasegawa, Correction to "On the convergence of resolvents of operators" 